On examination she had a fracture of the shaft of the right femur. There was no direct injury to the lower chest, abdomen, or pelvis. A few hours after admission she complained of upper abdominal discomfort and examination showed epigastric fullness. There was no rigidity or tenderness in the abdomen and bowel sounds were normal. The chest did not show any evidence of fractured ribs or haemopneumothorax. Plain $x$-ray of abdomen revealed gross distension of the stomach (see figure). The distension was treated with nasogastric suction and an intravenous drip. After 24 hours the abdominal discomfort and fullness disappeared and the patient was passing flatus normally. Her subsequent progress in a Thoma splint for the fractured femur was uneventful.

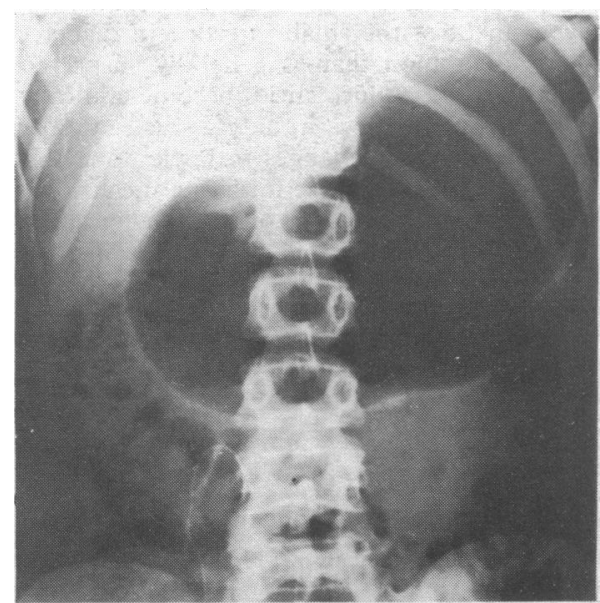

This case illustrates the fact that acute gastric dilatation can occur after trauma other than blunt injuries to the abdomen and, if there is no intra-abdominal injury, responds to intragastric suction. If this is borne in mind unnecessary laparotomy may be avoided.

S U RAHMAN

Burnley General Hospital, Burnley, Lancs

${ }^{1}$ Starr, K W, Annals of the Royal College of Surgeons of England, 1973, 12, 71

9th edn. Bristol, Wright, 1972 .
9th

\section{Effects of pethidine on the newborn}

SIR,-I must take issue with $\mathrm{Mr} \mathrm{J}$ Mair (14 August, p 425), who writes that the respiratory depressant effect of pethidine on the newborn has been overstated. He gives anecdotal evidence for his point of view by mentioning that "the condition and Apgar score of these babies have never been adversely affected by this regimen." The most relevant point he makes is that he administers $100 \mathrm{mg}$ of pethidine one hour before caesarean section. It is well known that pethidine given to mothers within one hour of delivery has little if any respiratory depressant effect on the newborn baby. ${ }^{12} \mathrm{His}$ conclusions are thus based on a series of cases in which one would not expect the problems of respiratory depression.

Your expert's answer to your correspondent (19 June, $p$ 1519) brings up some interesting questions. The lack of response to nalorphine, which is usually an effective antagonist to the respiratory depressant effect of pethidine, suggests that there may be another reason for this depression which occurs in neonates delivered to mothers who are given pethidine before delivery. It has been postulated that it may be the metabolites of pethidine ${ }^{2}$ which are the offending substances, norpethidine being known to be more toxic than pethidine. ${ }^{3}$ This may explain the lack of response to nalorphine in many instances. It may also explain the lack of respiratory depression found when pethidine is administered less than one hour before delivery.

There have been several recent papers suggesting that respiratory depression is bu one of several less obvious, though potentially harmful, effects of pethidine administration to mothers in labour. ${ }^{4-6}$

Paediatric Department,
Stoke Mandeville Hospital Aylesbury, Bucks

Shnider, S M, and Moya, F, American fournal of Obstetrics and Gynecology, 1964, 89, 1009.

Morrison, J C, et al, American fournal of Obstetrics and

Gynecology, 1973, 115, 1132 .
Miller, J W, and Anderson, H H, fournal of Pharma cology and Experimental Therapeutics, 1954, 112, 191 Richards, M P M, and Bernal, J F, in 3rd International
Congress on Obstetrics and Gynaecology, London, Congress on Obstetrics and Gyna

1971, P 303. Basel, Karger, 1972. Gynecology, 1974, 118, 377

'Desmond, M M, Anesthesiology, 1974, 40, 111

\section{Extrapyramidal side effects of lithium} treatment

SIR,-It is well known that various effects upon the extrapyramidal system may occur during treatment with neuroleptic drugs. Recently debate has arisen concerning extrapyramidal symptoms as an effect of lithium treatment. Shopsin and Gershon ${ }^{1}$ reported cogwheel rigidity in more than half of their patients under treatment with lithium for more than one year. The frequency was considered to increase with the duration of the treatment but was independent of age and of the dose given. However, in a later investigation Branchey et $a l^{2}$ were unable to confirm the high prevalence figures reported by Shopsin and Gershon.

Up to the present Parkinsonism as a complication of lithium treatment has been reported only occasionally in the literature and we therefore wish to draw attention to this phenomenon by describing two cases under our own care. An added reason is that the cause of these symptoms may easily be overlooked.

Case 1-A woman aged 57 years had been under reatment for nine years with lithium carbonate for a bipolar manic-depressive psychosis. Three weeks after an appendicectomy she was admitted to hospital because of anxiety and insomnia. On admission neurological examination revealed severe rigidity, stiffness of the neck musculature, dysarthria, dysphagia, and cogwheel rigidity of moderate degree in the limbs. In addition there was a suggestion of hypokinesia with only sligh tremor. At the time of admission the serum lithium level was $1.2 \mathrm{mmol} / 1(0.8 \mathrm{mg} / 100 \mathrm{ml})$. After the lithium had been discontinued the symptoms subsided over a period of two weeks. Subsequently she has remained well and completely free from extrapyramidal symptoms on a maintenance dosage of lithium lower than the previous one.

Case 2-A man 59 years of age suffering from bipolar manic-depressive psychosis had been under treatment with lithium for seven years. He had also received neuroleptics and tricyclic antidepressives for periods. Extrapyramidal symptoms had not previously been observed. He was admitted to hospital because of lassitude and attacks of diarrhoea. On admission there was some impairment of alertness, slurred speech, and unsteady gait. Before admission, in addition to lithium carbonate, he had been kept on haloperidol 2-3 mg daily. Both drugs were discontinued on the day of admission. During the first few days after this he developed a Parkinson-like picture with pronounced rigidity of cogwheel type, principally in the neck and arms, forward-leaning gait, shor steps without related movements, and hypersalivation, but no tremor. The initial serum lithium level was $1.9 \mathrm{mmol} / 1(1.3 \mathrm{mg} / 100 \mathrm{ml})$. The symptoms regressed completely over a period of about two weeks. It was subsequently possible to restart haloperidol and lithium in reduced dosage without any recurrence of the extrapyramidal symptoms.

Earlier descriptions ${ }^{3} 4$ of patients with lithium intoxication also include symptoms of extrapyramidal type, to some extent resembling those we have observed. In our two patients, both under treatment with lithium for many years and in whom similar symptoms had not previously been observed, moderately raised serum lithium concentrations were associated with severe rigidity, most evident in the rostral musculature. Stiffness in the face and neck, difficulty in swallowing, and dysarthria were the most prominent features. The limbs showed the cogwheel phenomenon. In both cases the element of tremor was inconspicuous, but there was slight hypokinesia. This picture of extrapyramidal symptoms thus departs in many respects from that of Parkinson's disease and that seen in neuroleptic treatment.

Dalén and Steg ${ }^{5}$ observed that lithium reduces hyperkinetic symptoms both in choreic patients and in those treated with levodopa for Parkinson's disease and also that it has a tendency to aggravate the symptoms of Parkinsonism.

The occurrence of extrapyramidal symptoms in connection with lithium treatment may, at least in part, be due to an inhibition of transmission in the dopaminergic conduction system in the central nervous system. This effect might well include a reduction of the sensitivity of the dopamine receptors. In our view there is as yet no definite proof that the observed extrapyramidal symptoms are dependent on the duration of the treatment, but attention should be directed to this question. It may be that some underlying or predisposing cerebral dysfunction is a necessary condition for the appearance of these symptoms.

BO JOHNELS

University of Göteborg,

Department of Neurology,

Sahlgren's Hospital,

Göteborg, Sweden

LEIF WALLIN

JAN WÅLINDER

University of Göteborg,

St Jörgen Hospital,

Hisings Backa, Sweden
Hisit

Shopsin, B, and Gershon, S, American fournal of Psychiatry, 1975, 132, 536.

Branchey, M H, Charles, J, and Simpson, G M, American fournal of Psychiatry, 1976, 133, 44 Stern, R L, Fournal of the American Medical Associa-

tion, 1949, 139, 710.
- van der Velde, C D, American fournal of Psychiatry,

s Dalén, P, and Steg, G, Lancet, 1973, 1, 936.

\section{Pyloric stenosis and sustained-release} iron tablets

SIR,-The case reported below gives an interesting and salutary warning about the possible effects of giving long-acting iron tablets to patients with a history of peptic ulceration or long-standing dyspepsia.

The patient, an arthritic spinster of 74 who was known to live on a diet of cake and Maltesers, was started on treatment with Ferrogradumet $\mathrm{C}$ for a 
dietary anaemia. She previously had a history of vague epigastric pain after meals. Her condition generally deteriorated and nausea and anorexia became predominant symptoms. Four days after admission to a geriatric unit she vomited 70 plastic matrices of her Ferrogradumet $C$ tablets, with immediate relief of her symptoms.

One can only assume that in patients with delayed gastric emptying a situation of pyloric stenosis can be brought about by the administration of this type of tablet. The mechanism is presumably accumulation of the "honeycombed" plastic matrices, through which the active drug, together with a watersoluble channelling agent, passes. In cases in which there is a narrowed or constricted stomach outlet one should perhaps be cautious in the use of such delayed-release iron tablets.

Incidentally, in normal circumstances, the filtration process occurs in the upper small intestine, with the ultimate excretion of the matrix, empty, in the stools.

\section{P CARNE-Ross}

Meopham, Kent

\section{A hazard of apple-eating}

SIR,-An apple a day doesn't always keep the doctor away, as can be learnt from the following case.

A 55-year-old woman was referred to our department for neurological examination. She had a history lasting more than 20 years of attacks of dizziness and syncope, mostly in association with meals. The first time she had fainted was believed to be in connection with a delivery about 25 years previously; this had been regarded as some sort of epileptic attack. However, convulsions had never been observed.

During recent years the attacks had become more alarming, with frequent fainting, principally when swallowing in the sitting position. Further questioning showed that apples and strawberries were most likely to provoke the attacks, which consisted of periods of unconsciousness lasting not longer than 30 seconds, still without convulsions. Previous $x$-ray of the oesophagus was normal. The electrocardiogram (ECG) at rest was normal. Electroencephalography had shown a suspected paroxysmal activity. Complete neurological examination was normal.

Because of the history an ECG was registered while the patient was eating an apple. During the act of swallowing the patient had a feeling of dizziness, and an irregular pulse was palpated, but she did not faint. The ECG showed that the patient had a grade -3 atrioventricular block (see figure) which disappeared spontanously after swallowing had ceased. Repeated experiments with apple-eating gave the same results even when the patient had chewed the pieces carefully. Other fruits, such as oranges and pears, gave no reaction, nor did potatoes or bread and butter. Strawberries could not be tried because of the season.

The patient was given hyoscyamine sulphate in small doses, which resulted in a feeling of well- being and safety and she was able to eat apples again. The only necessary treatment would of course have been to exclude apples from the diet.

This case illustrates how easy it is to mistake Adams-Stokes attacks for some sort of neurological disorder, most frequently epilepsy. A wide survey of the literature on this subject has been published by Johansson. ${ }^{1}$ Our case shows that Adams-Stokes attacks may be provoked by vagal stimuli only in special situations. It would have been tempting to assume that the pieces of apple provoked the attacks by a mechanical effect. However, the attacks were noted even after careful chewing and were not provoked by fairly large lumps of potatoes or bread. It may be speculated whether some sort of chemical stimulation of the oesophagus was also necessary.

\section{ThOMas HaVland} GÖRAN FRITHZ

Department of Medicine,

\section{Central Hospital,} 1 Johansson, B W, American fournal of Cardiology,
1961, 7, 874.

\section{Doctors, contraception, and} sterilisation

SIR,-In reply to Dr P W Lambden's letter (28 August, p 528) I agree that the duty of the family doctor is to give total patient care on the prevention and treatment of disease and injury, which also includes advice on contraception. Of course I am in favour of responsible family planning, but I am concerned about some of the early and late results of introducing either synthetic or natural hormones into a normally functioning feminine physiological cycle.

It is recognised that our business is to see that "the drug does no harm" and "the pill" has been shown to cause venous thrombosis and embolism and, more recently, to be associated with vascular tumours in the liver, one of which has been found to be undergoing malignant change. Knowing this, should we not pause for thought and advise treatment which carries no risk to the patient or her offspring should she, after a space of time, wish to have another child?

As for vasectomy, I regret that Dr Lambden seems to have misunderstood my use of the word "mutilation." Surely the excision of a section of the vasa in a normal healthy male, though expertly and neatly carried out, is a mutilation.

There are other means of contraception than by medical or surgical procedures but demanding responsibility on the part of one or both partners.

Ipswich, Suffolk
National Health Service Consultants' Association

SIR,-The National Health Service Consultants' Association was formed in early 1976 following an enthusiastic response to a letter which appeared in the Lancet $^{1}$

Our basic concern is to save and improve the NHS. We are committed to its basic philosophy of providing medical care to those in need regardless of their social or financial status. Inevitably we are critical of certain aspects of the administration of the NHS, but we are increasingly concerned about the damaging effects on the service (which really means on sick people) of industrial action by doctors in pursuit of their own financial interests. In general we feel that the NHS is best served by a whole-time commitment and that there should be appropriate inducements, financial and otherwise, for the vast majority of consultants to wish to make this commitment. We do not feel that existing bodies such as the BMA and Hospital Consultants and Specialists Association adequately represent this point of view to the DHSS or to the public.

As at least half of consultants are whole-time we feel that there must be many of our colleagues who will be sympathetic with the general tenor of the views outlined above.

The association held its inaugural meeting on 20 May 1976 and since then we have been collecting evidence from our members for submission to the Royal Commission on the NHS as we feel it most important that the whole-timers' view should be adequately represented to this body-certainly the views of those consultants who are fully committed to the NHS seems to have been underrepresented in the disputes about pay-beds and about contracts.

I will be happy to send further information about the association and its aims to those of your readers who might be interested in joining us.

SAM BAXTER

National Health Service Consultants' Association o Department of Psychiatry,

Charing Cross Hospital,
London W6

${ }^{1}$ Noone, P, et al, Lancet, 1976, 1, 141.

\section{Consultants' contract}

SIR,-It is apparent from listening to the debates on consultant contracts that the consultant body does not have a clear idea on which to base plans for a new contract. I would like to remedy this deficit by suggesting a contract which combines the advantages of the whole-time contract with those of the part-time contract and introduces a degree of flexibility which it is obvious that consultants desire.

The basic commitment would be to a number of hours' working time during which the consultant would be available for work in hospital or elsewhere. This would be assessed for each consultant and, for the average wholetime consultant, would probably amount to between 40 and 50 hours if the evidence given to the Owen working party is still applicable. Part-time consultants would be able to contract for a lesser amount of "available working time" (AWT). The lower limit of AWTs might be requested by the employing authority and could reasonably be negotiated between 25 and 30 hours. The upper limit of AWTs could be 